\title{
The Influence of Efficiency on Receive Diversity and MIMO Capacity for Rayleigh-Fading Channels
}

\author{
Juan F. Valenzuela-Valdés, Miguel A. García-Fernández, Antonio M. Martínez-González, and \\ David A. Sánchez-Hernández, Senior Member, IEEE
}

\begin{abstract}
A previously published guideline for MIMO antenna arrays is refuted. The influence of radiation efficiency on diversity gain and MIMO capacity of wireless communications systems is investigated through simulations and measurements using a reverberation chamber. Integrated antennas on a portable device have efficiencies low enough to disallow typical inter-element correlation assumptions. Both diversity gain and MIMO capacity depend on the number of antennas, SNR and efficiency in a complex way. When the efficiency of antennas is considered, certain system capacity losses are predicted and measured. These losses may be recovered through using more receive elements than commonly recommended or through the addition of a smaller number of more efficient antennas.
\end{abstract}

Index Terms-Diversity gain, channel capacity, multiple-inputmultiple-output (MIMO) systems, radiation efficiency.

\section{INTRODUCTION}

$\mathbf{M}$ ANY STUDIES have been carried out to optimize the maximum capacity attained with multiple-input multiple-output (MIMO) systems. This is typically done through the achievement of high diversity gain, which requires a low correlation between the diversity branches. The diverse factors affecting diversity gain [1]-[4] and MIMO capacity [5], [6] have been deeply but independently studied in the literature, with the aim of reducing the correlation coefficients between signals. Spatial [7], [8], angular [9], [10], pattern [11] or a combination of these [12] have been reported. In these studies, however, ideal radiation efficiencies are typically employed. In practice, handset antenna efficiencies are low [13], [14]. Likewise, it has been assumed for some time now that there is no need to use more receiving antennas $(R)$ than transmitting antennas $(T)$ [6], [7]. This is due to a predicted minuscule capacity improvement, but yet under noise-limiting conditions and very large power angular spread $\left(\sim 360^{\circ}\right)$, which may not be assumed in the presence of the user. Consequently, there is need to investigate whether radiation efficiency can

Manuscript received October 3, 2006; revised January 22, 2008. This work was supported in part by the Fundación Séneca, the R\&D unit of the Autonomous Region of Murcia (Spain) under project references TIC-TEC 06/01-0003, 07/02-0005 and 05746/PI/07, and in part by the Spanish National R\&D Programme through TEC2007/63470/TCM.

J. F. Valenzuela-Valdés is with the EMITE Ingeniería SLNE, Edificio CEEIM, Campus Espinardo, E-30100 Espinardo, Murcia, Spain (e-mail: juan.valenzuela@emite-ingenieria.es).

M. A. García-Fernández, A. M. Martínez-González, and D. A. SánchezHernández are with the Departamento de Tecnologías de la Información y Comunicaciones, Universidad Politécnica de Cartagena, Cartagena E-30202, Spain (e-mail: ddavid.sanchez@upct.es).

Digital Object Identifier 10.1109/TAP.2008.922208 significantly reduce the potential of novel MIMO systems in the close vicinity of the user. This is reinforced by the fact that the importance of radiation efficiency in [15] also outlined large theoretical inaccuracies and was only studied through simulations for a 2-branch diversity scheme within isotropic signal environments. In summary, the intense MIMO research activity has prompted new issues related to the impact of antenna properties on MIMO performance. With the novel MIMO systems being proposed for the handset scenario in close proximity to the user, it becomes essential to improve current knowledge on the effects of efficiency on MIMO system performance. This will in turn be useful for predicting whether viable uncorrelated MIMO channels can be expected for these new scenarios, and can possibly bring light to the still-unknown best array topology in terms of maximizing capacity [16].

In this paper we evaluate the complex role of radiation efficiency on both effective diversity gain and MIMO capacity for MIMO channels through both simulations and measurements in a reverberation chamber. The antennas were measured individually so that unused antennas are omitted from the structure. This was done to avoid correlation due to mutual coupling and for the evaluation of only efficiency-related effects.

\section{Theoretical Model AND Simulated Results}

\section{A. Receive Diversity and MIMO Performance}

While diversity has received considerable attention in the literature, few studies tackle receive diversity measurements in the presence of the user and thereby reduced radiation efficiencies. This has been identified as still an open issue [4], [14]. The radiated power in this scenario is the power available for communication. It can be determined by integrating the normal component of the time-averaged Poynting vector over a closed surface, which encloses the antenna and the user. The radiated power $P_{\text {rad }}$ can also be defined as,

$$
\begin{aligned}
P_{\mathrm{rad}} & =P_{\mathrm{max}}-P_{\mathrm{tloss}} \\
& =P_{\mathrm{av}}-P_{\mathrm{diss}}-P_{\mathrm{body}}-P_{\mathrm{emb}}-P_{m}
\end{aligned}
$$

where

$P_{\max } \quad$ maximum power available at the antenna input;

$P_{\text {tloss }}$ total power loss;

$P_{\text {diss }} \quad$ ohmic and dielectric power loss; 
$P_{\text {body }}$ power absorbed in the human body;

$P_{\mathrm{emb}} \quad$ power dissipated in other antennas through mutual coupling;

$P_{\mathrm{m}} \quad$ power loss due to impedance mismatch.

Significant gain reduction occurs when an antenna is used close to the human body, with resulting radiation efficiencies below 50\% [14]. When the antenna is integrated in a small volume, an additional efficiency reduction is also expected [17], aggravating potential MIMO performance. The user presence at the receiver end also affects MIMO performance by increasing the correlation coefficients when the head blocks the signals, diminishing dissimilarities in the radiation patterns away from the head [18], [19]. Consequently, a proper study of the effect of radiation efficiency on MIMO performance must include both diversity gain and capacity.

\section{B. Total Radiation Efficiency}

The total radiation efficiency $\eta_{\mathrm{ef}}$ is the ratio between the radiated power $P_{\text {rad }}$ and the total available power

$$
\eta_{\mathrm{ef}}=\frac{P_{\mathrm{rad}}}{P_{\mathrm{rad}}+P_{\mathrm{body}}+P_{\mathrm{diss}}+P_{\mathrm{emb}}+P_{m}}
$$

Mutual coupling reduces efficiency and the mean effective gain (MEG) [4], [20], and can be accounted for the total embedded radiation efficiency [14]. Alternatively, accounting for mutual coupling can be eluded since for wireless communications it is not constant and depends strongly on multipath and scattering objects [21]. In order to aid clarity and identify efficiency-related effects only, mutual coupling has not been considered in this study $\left(P_{\mathrm{emb}}=0\right)$ for the calculation of the total radiation efficiency, in a similar way to recent switched array techniques [21].

\section{Diversity Gain}

Several definitions of the diversity gain are available depending upon the selected reference. For the apparent diversity gain (ADG) the reference is the strongest branch/antenna while for the effective diversity gain (EDG) the reference is an antenna with $100 \%$ efficiency. This reference is obtained by using an antenna with known efficiency and correcting for its known efficiency [22]. In order to identify only efficiency-related effects, we have defined the ideal diversity gain (IDG), where the reference is the theoretical upperbound Rayleigh curve and measurements are taken for isolated antennas so as to avoid mutual coupling. While a few studies dealt with efficiency-related effects on diversity gain [13], [23], [24], available studies on MIMO capacity do not include the effects of radiation efficiency. Furthermore, contradictory findings can be found for the effect of radiation efficiency on diversity gain. A reduction in efficiency with user presence did translate in a large increase in the MEG for several whip and PIFA antennas in [14], but the contrary (MEG was halved) was found in [18] for several vertical and horizontal monopole and PIFA antennas.

In order to calculate EDG, the radiation efficiency for the reference antenna $\eta_{\text {ref }}$ has to be known, while this is not the case for the IDG. For EDG measurements the antenna noise temperature

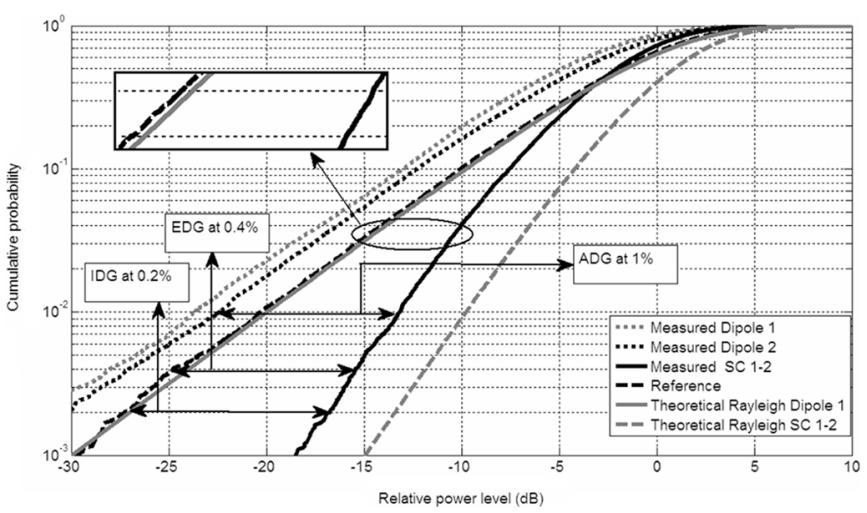

Fig. 1. CDFs versus relative received power level (dB) for two orthogonal $\lambda / 2$ dipoles with $\eta_{1}=0.51$ and $\eta_{2}=0.59$ in the reverberation chamber.

is assumed to be approximately equal to the ambient temperature. This is in turn linked to no dominant line-of-sight (LoS) scenarios typically used in reverberation chambers to emulate outdoor Rayleigh-fading environments. EDG is inherently less accurate than IDG since it depends upon the efficiency of the antenna reference, which usually has an uncertainty component. With the introduction of IDG another term can immediately be coined for the employed combining scheme of the $N$ receiving antennas; the diversity gain loss (DGL) as

$$
\mathrm{DGL}_{N}(\mathrm{~dB})=\operatorname{IDG}(\mathrm{dB})-\operatorname{comb}_{N}(\mathrm{~dB})
$$

where $\operatorname{comb}_{N}$ is the combined effect of radiation efficiencies of the $N$ receiving antennas. If the signals are uncorrelated, this new term also can be defined as

$$
\mathrm{DGL}_{N}(\mathrm{~dB})=-\left(\sum_{i=1}^{i=N} \eta_{i}(\mathrm{~dB})\right) / N
$$

where $\eta_{i}$ is the radiation efficiency of the $i$ th antenna and $N$ is the total number of receiving antennas. Fig. 1 and Table I illustrate the concepts of ADG, EDG, IDG and DGL for a twobranch diversity scheme using $\lambda / 2$ dipoles in the reverberation chamber. From Fig. 1 we can observe that the effect of efficiency on the cumulative probability density functions (CDF) is a displacement of the curve to the left, but the slope is not affected. In order to evaluate the effect of the total radiation efficiency on diversity gain, we can write

$$
\mathrm{SNR}_{\mathrm{we}}(\mathrm{dB})=\mathrm{SNR}(\mathrm{dB})+\eta_{\mathrm{ef}}(\mathrm{dB})
$$

where $\mathrm{SNR}_{\mathrm{we}}$ is the signal to noise ratio with the efficiency effect included.

\section{MIMO Capacity}

The instantaneous channel capacity for MIMO systems is well known, and can be defined to account for efficiency by

$$
\begin{aligned}
& \text { C.MIMO }_{\mathrm{we}}=\log _{2} \\
& \qquad\left(\operatorname{det}\left(I_{R}+\frac{\mathrm{SNR}_{\mathrm{we}}}{T} * H * H^{\prime}\right)\right) \mathrm{bits} / \mathrm{s} / \mathrm{Hz}
\end{aligned}
$$


TABLE I

COMPARISON OF DIFFERENT DIVERSITY GAIN DEFINITIONS

\begin{tabular}{|l|c|c|c|}
\hline & EDG $(\mathrm{dB})$ & IDG $(\mathrm{dB})$ & ADG $(\mathrm{dB})$ \\
\hline At $1 \%$ & -7.00 & -6.83 & -9.28 \\
\hline At $0.5 \%$ & -9.01 & -8.82 & -11.77 \\
\hline At $0.3 \%$ & -9.47 & -9.22 & -12.49 \\
\hline At $0.1 \%$ & -11.36 & -11.52 & -15.12 \\
\hline
\end{tabular}

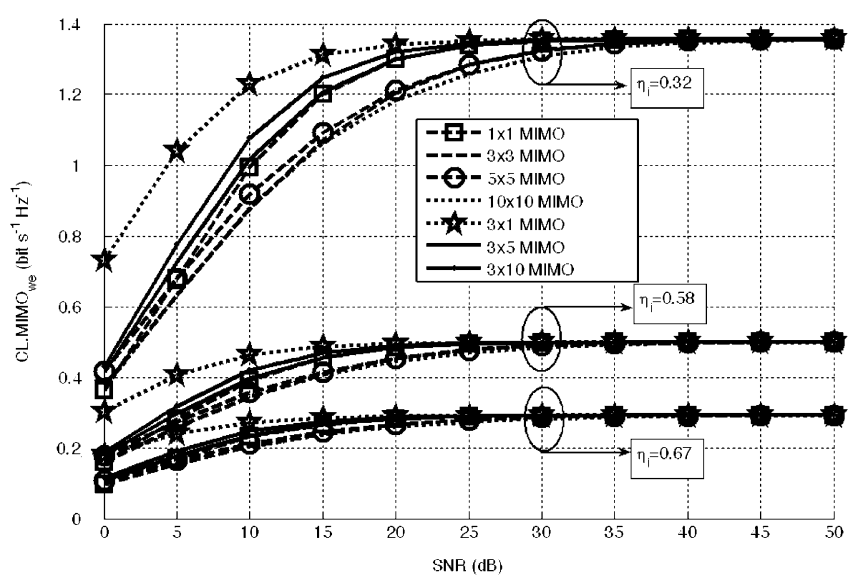

Fig. 2. Simulated MIMO capacity loss versus SNR for different systems.

with $T$ antennas at the transmitter, $R$ antennas at the receiver and $I_{R}$ as the identity matrix with dimension $R$. In an independent identically distributed (i.i.d.) [25], [26] Rayleigh environment this capacity can be approximated for high signal-to-noise ratio (SNR) to [6]

$$
\text { C.MIMO }_{\text {we }}=\min (T, R) \log _{2}\left(\mathrm{SNR}_{\mathrm{we}}\right) \text { bits } / \mathrm{s} / \mathrm{Hz} .
$$

We can extract the radiation efficiency effect in (7) for high SNRs by defining the MIMO capacity loss due to efficiency $\left(\right.$ CL.MIMO $\left._{\text {we }}\right)$ as

$$
\text { CL.MIMO }{ }_{\text {we }}=-\min (T, R) \log _{2}\left(\eta_{\text {ef }}\right) \quad \text { bits } / \mathrm{s} / \mathrm{Hz} .
$$

For low SNRs, the dependence of MIMO capacity on radiation efficiency is not straightforward, and therefore (6) was used for the simulations. In Fig. 2 we show simulated MIMO capacity loss due to a diverse common radiation efficiencies $\eta_{i}$ with different numbers of branches/antennas versus SNR. MIMO capacity loss is depicted per fully parallel channel, that is, divided by the minimum of $T$ and $R$. Ninety percent of the capacity loss due to radiation efficiency is reached in all studied systems for a $\mathrm{SNR}=15 \mathrm{~dB}$. Lower efficiencies require higher SNRs for achieving $90 \%$ of the capacity loss due to radiation efficiency. From these simulations it seems clear that efficiency plays an important role for low SNRs since capacity loss is practically constant above SNR $=35 \mathrm{~dB}$ for all combinations.

Fig. 3 depicts simulated MIMO capacity loss due to radiation efficiency for different systems with two different SNR (5 and 20 $\mathrm{dB}), T=3$ and $R$ as parameter. MIMO capacity loss due to efficiency increases linearly with increasing number of receiving antennas until $T=R$, where a $90 \%$ of its maximum is reached. Capacity loss reaches $90 \%$ of its maximum when $R=T$ for all studied systems, making additional capacity loss due to efficiency for $R>T$ MIMO systems negligible compared to $R=T$

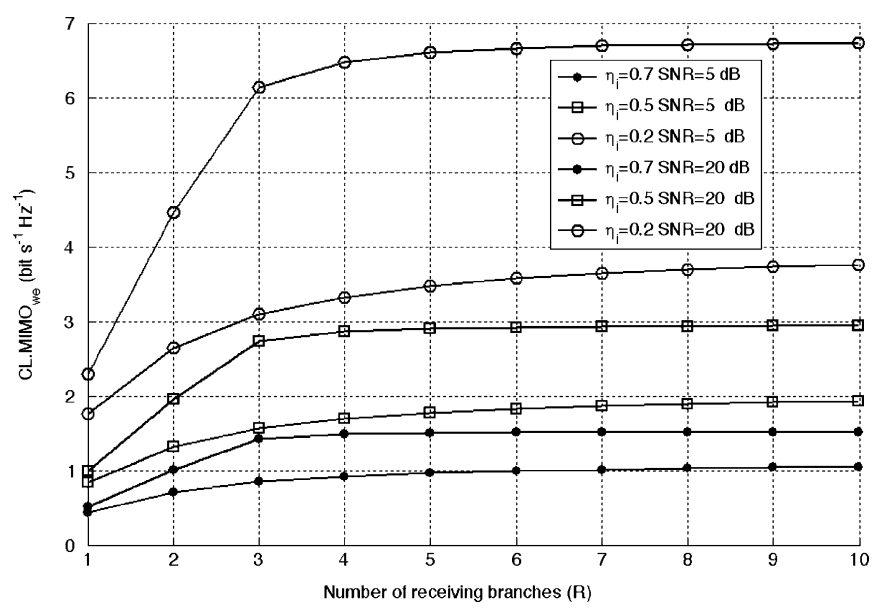

Fig. 3. Simulated MIMO capacity loss for different radiation efficiencies versus $\mathrm{R}$ with $\mathrm{T}=3$.

MIMO systems. Consequently, adding a highly efficient antenna may enhance final MIMO capacity when radiation efficiencies are accounted for, which always happens in practice.

\section{MEAsurement SET-UP AND RESUlts}

The results obtained in the simulations were validated by measurements in the Bluetest AB RC800 reverberation chamber. Measurements were performed at different frequencies with commercial $\lambda / 2$ Bluetest 001-B-019 dipoles with operating ranges from 890 to $3000 \mathrm{MHz}$. In order to evaluate only the influence of radiation efficiencies on diversity gain and MIMO capacity we have measured each one of 10 isolated antennas in a different position within the reverberation chamber, that is, using uncorrelated branches with the same received power level but different radiation efficiencies for the MIMO system under evaluation. With this measurement set-up we eliminated the correlation between signals due to mutual coupling, thus reproducing the theoretical model described in Section II. A full description of the measuring chamber and radiation efficiency measurement technique can be found in [22], [23] and [24], respectively. In order to be able to measure the MIMO potential of antennas with different efficiencies in the handset scenario, that is, in the presence of the user, a 32.3 $\mathrm{cm}$ high and $12.5 \mathrm{~cm}$ in diameter lossy cylinder filled with CENELEC A2400 head simulating liquid (HSL) was employed within the reverberation chamber. Diverse radiation efficiencies from 0.32 to 0.86 were obtained by locating the lossy cylinder at different relative positions respect to the antenna under evaluation [27].

\section{A. Diversity Gain}

The effective diversity gain (EDG) is obtained by collecting received power samples over a $20 \mathrm{MHz}$ bandwidth, with a 0.1 $\mathrm{MHz}$ frequency steps. Each frequency point consists of 50 stirrer positions, providing 10050 independent power samples for each measured antenna so that a rich Rayleigh-fading environment was ensured. Fig. 4 illustrates measured EDG for simple combining situations of up to ten equally-efficient receiving $\lambda / 2$ dipoles. From this figure it is confirmed that increasing the number of receiving antennas has a positive 


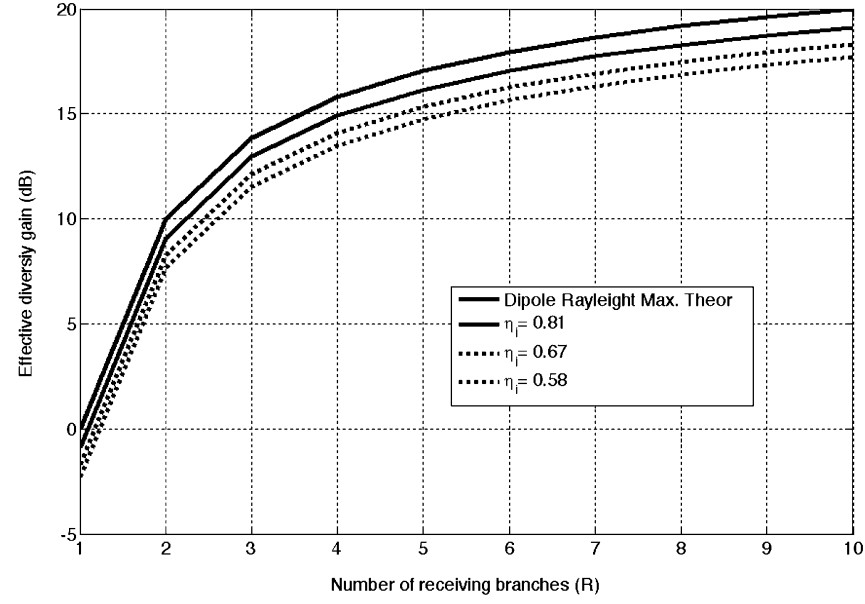

Fig. 4. Measured EDG versus R receive antennas for different efficiencies.

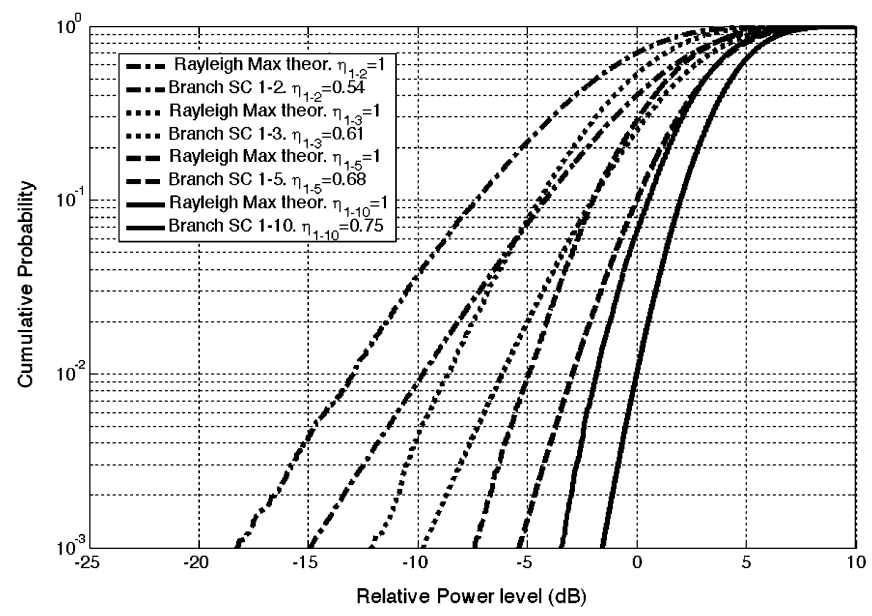

Fig. 5. Measured CDFs for different MIMO systems.

effect on EDG, but from $R=T$ onwards the increment rate slows down considerably. For a $T=3$ system with antenna efficiencies of 0.36 , increasing the number of receiving antennas from 2 to 3 attains an additional $5 \mathrm{~dB}$ in EDG. This is exactly the same additional EDG that can be obtained when adding 7 extra antennas to the $R=3$ situation. Better results, however, are observed when adding more efficient antennas. The same $T=3$ system with $\eta_{i}=0.81$ attains $4 \mathrm{~dB}$ extra EDG when adding an additional antenna to an $R=2$ system, but $\sim 6 \mathrm{~dB}$ are gained when 7 antennas are added to the $R=3$ situation. Consequently, efficiency does play an effect on the increasing EDG, apart from simply providing worse final EDG values compared to ideal lossless antennas, even when all receiving antennas exhibit the same radiation efficiency. Fig. 5 shows different measured combining scenarios. Measurements agree well with the simulations.

\section{B. MIMO Capacity}

Channel capacity is calculated using the measured channel estimates between each of the $n$ MIMO receiving antennas and each one of the $m$ wall-mounted transmitting exciting antennas [24]. All channel capacity estimates are averaged to produce a maximum average channel capacity as a function of the SNR,

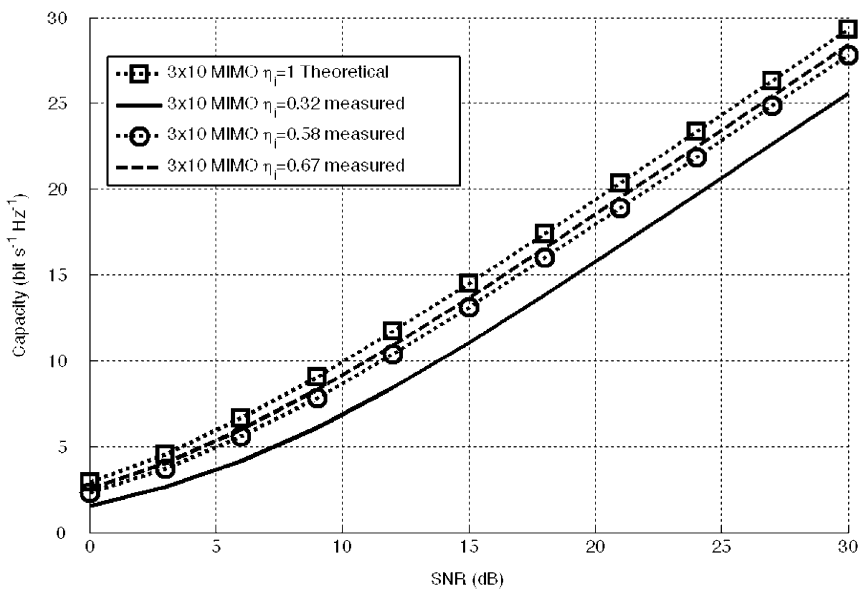

Fig. 6. Measured MIMO capacity versus SNR for different total radiation efficiencies.

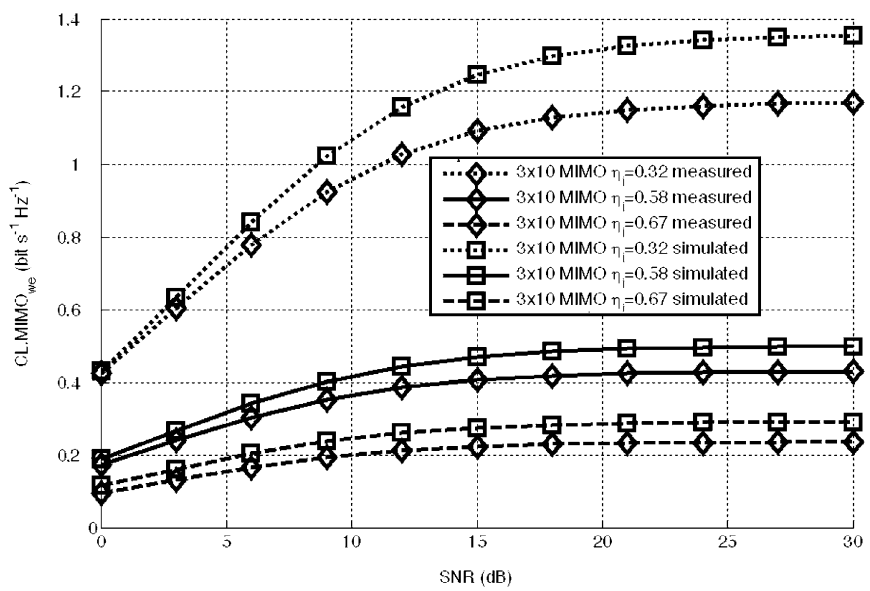

Fig. 7. Measured and simulated capacity loss due to efficiency versus SNR for diverse total radiation efficiencies in $3 \times 10$ MIMO systems.

identified as mean capacity [24]. To validate the decrease in MIMO capacity obtained in the simulations when real efficiencies are employed, Figs. 6 and 7 exhibit MIMO capacity and capacity loss due to efficiency, respectively, for four different MIMO systems with the same radiation efficiency for all receiving antennas, but different total radiation efficiency for each system. From these figures we can observe that the simulated results in Section II are validated. It is also clear from these figures that efficiency plays an important role when determining MIMO capacity, particularly at low SNR values. With a SNR of $15 \mathrm{~dB}, 25 \%$ capacity loss can be expected when low efficient antennas are employed instead of highly efficient antennas, or simply when the user is present in close proximity to the MIMO receiving antennas. Consequently, the combining possibilities and antenna topology for MIMO systems in the presence of the user acquires great importance. At $\mathrm{SNR}=10 \mathrm{~dB}$, a maximum of $1.6 \%$ percentage error was observed between simulated and measured MIMO capacity results.

In this sense, Fig. 8 reproduces measured results of capacity loss due to efficiency for different $3 \times n$ MIMO systems with the same radiation efficiency for all antennas in one branch. While there is always a capacity loss, this is more clearly appreciated when the receiving antennas exhibit a low efficiency. Likewise, it is also observed that the additional capacity loss 


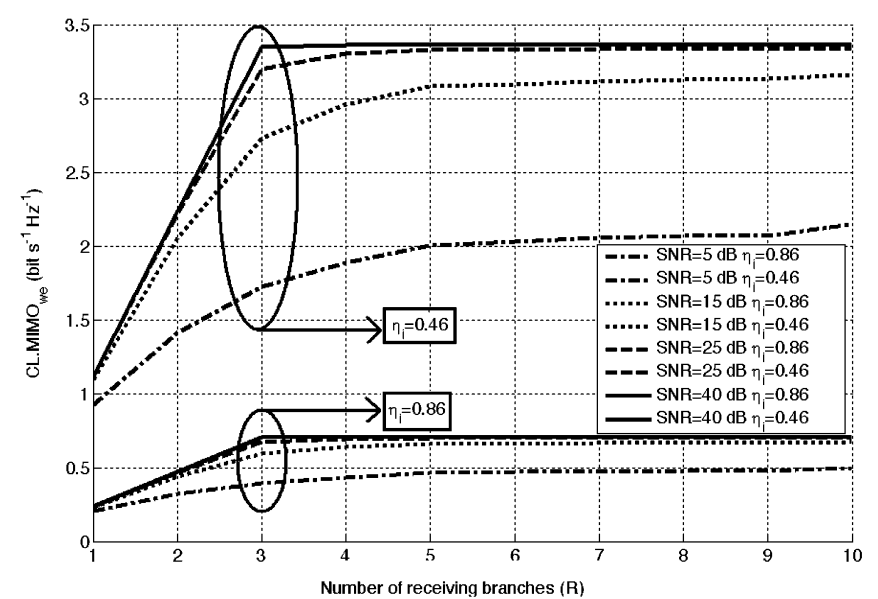

Fig. 8. Measured MIMO capacity loss due to efficiency with $\mathrm{T}=3$ and $\mathrm{R}$ as parameter.

obtained when $R>T$ and employed receiving antennas are inefficient is mitigated when high SNRs values are obtained, confirming the simulated results presented in Section II. Similarly, the effect is more important when $R \leq T$, with a high slope that stabilizes when $R=T$ only for high SNRs. On the other hand, a quasi-linear increase with increasing $R$ in MIMO capacity loss due to efficiency is observed when SNR is low. Therefore, the effect of efficiency on MIMO capacity acquires great importance for low efficient antennas and low SNRs.

It is highly unlikely, however, that all receiving antennas have the same radiation efficiency despite the limited volume available at the handset scenario, not only due to the different antenna-user distances, but also because each antenna design and topology may exhibit different radiation properties [4]. At the same time, it has been assumed for some time now that under noise-limiting conditions and despite theoretical MIMO erdogic capacity formulas, there is little incentive on using more receiving antennas than transmitting antennas [6], that is, $\beta=$ $\mathrm{T} / \mathrm{R}<1$. Yet, this widely accepted formula assumes that there is an area of local scattering around each terminal and a very large power angular spread $\left(\sim 360^{\circ}\right)$. The presence of the user for receive diversity increases local scattering, but considerably reduces angular spread [14], therefore an effect of the total radiation efficiency on the validity of this formula is expected. Fig. 9 illustrates the simulated and measured effect of adding high efficiency transmitting or receiving antennas to a $3 \times 3 \mathrm{MIMO}$ system, initially comprising low efficiency receiving antennas. A good matching between simulated and measured results is again observed. As expected, the increment in MIMO capacity is clear when both transmitting and receiving antennas are simultaneously added to the system, even when these added antennas have a low efficiency. Yet, it is interesting to observe from this figure that, unlike what is widely accepted, there is a non-negligible capacity increase beyond $R=T$ when high efficiency antennas are added to a low efficient MIMO system. Likewise, this increment beyond $R=T$ is more important as SNR increases. Adding a receiving antenna with an efficiency of 0.86 to a $3 \times 3 \mathrm{MIMO}$ system with $\eta_{i}=0.2$, for instance, provides the same MIMO capacity than a $4 \times 4$ MIMO system with $\eta_{i}=0.46$ at $\mathrm{SNR}=20 \mathrm{~dB}$ or a $4 \times 4 \mathrm{MIMO}$ system with

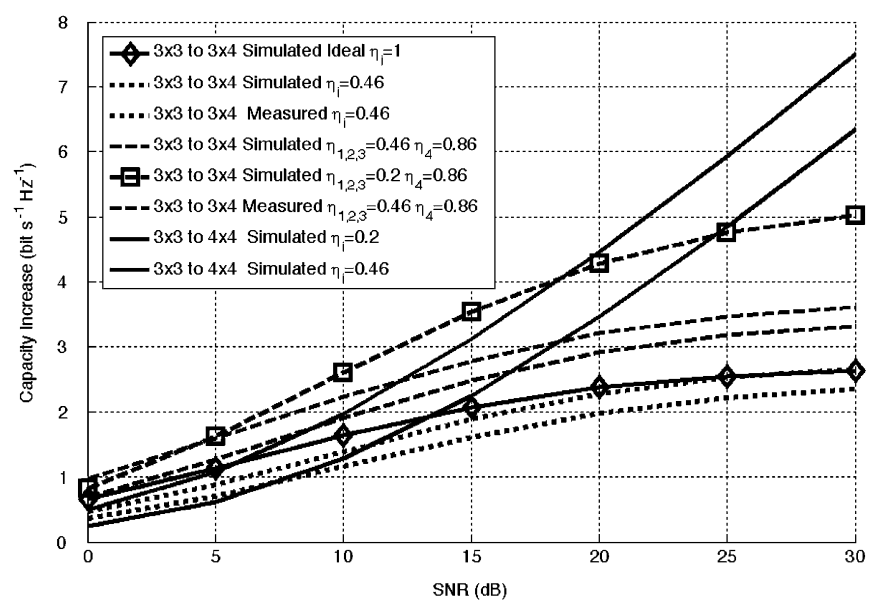

Fig. 9. Measured capacity increase when adding antennas to a $3 \times 3$ MIMO system. The figure shows how capacity climbs as antennas are added with eventual diminishing returns. For a MIMO system operating at low SNR $(<18 \mathrm{~dB})$, $M$ efficient receive antennas can achieve higher capacity than $M+1$ less efficient ones.

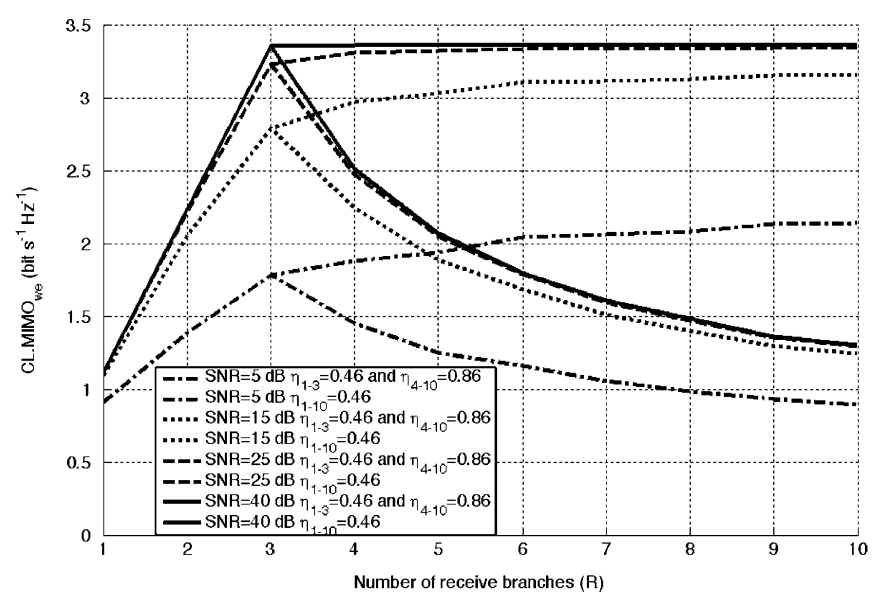

Fig. 10. Measured capacity loss due to efficiency for MIMO systems with $\mathrm{T}=$ 3 and $\mathrm{R}$ as parameter.

$\eta_{i}=0.2$ at $\mathrm{SNR}=25 \mathrm{~dB}$. In fact, Fig. 9 also shows that just adding a high efficiency receiving antenna to the $3 \times 3 \mathrm{MIMO}$ system is better in terms of capacity increase than adding both a receiving and a transmitting low efficient antenna, but only for a specific SNR value that depends on the radiation efficiencies of the added antennas. With $\eta_{4}=0.86$ in a $3 \times 4$ MIMO system instead of $\eta_{4}=0.46$ in a $4 \times 4$ MIMO system, this limiting value is $\mathrm{SNR}=18.45 \mathrm{~dB}$.

This increment in capacity is also clearly observed from Fig. 10, wherein the capacity loss due to efficiency is reduced when $R>T(\beta<1)$ but only when the added antennas have a high efficiency. It can also be concluded from Fig. 10 that the capacity increase beyond $\beta=1$, or the equivalent reduction in capacity loss due to efficiency, is only important at low and moderate SNRs. No significant improvement is experimented between results at $\mathrm{SNR}=25 \mathrm{~dB}$ and those at $\mathrm{SNR}=40 \mathrm{~dB}$.

\section{CONCLUSION}

In this paper, we have evaluated the effect of radiation efficiency on diversity gain and MIMO capacity through simula- 
tions and measurements in a reverberation chamber. It has been demonstrated that both diversity gain and MIMO capacity depend upon the number of antennas, SNR and total radiation efficiency on a complex way. The main discovery associated to this dependence is directly related to the optimum number of receiving antennas in a specific MIMO scenario. While it has been assumed for some time now that there is little incentive on using more $R$ receiving antennas than $T$ transmitting antennas, in the presence of the user this is no longer true and it depends very much on the radiation efficiencies of the antennas themselves. With $\beta=\mathrm{T} / \mathrm{R}$ showing a much more complex optimum behavior than unity, results are particularly important for the novel recently proposed MIMO designs on handheld terminals. Future research includes the extension to other fading scenarios and the specific dependence of efficiency-related effects on the number of scatters (NS), angular spread (AS) and angle of arrival (AoA), among others.

\section{ACKNOWLEDGMENT}

The authors wish to thank the Autonomous Region of Murcia, Spain, for partly funding this research.

\section{REFERENCES}

[1] A. M. D. Turkmani, A. A. Arowojolu, P. A. Jefford, and C. J. Kellett, "An experimental evaluation of the performance two-branch space and polarization diversity schemes at $1800 \mathrm{MHz}, "$ IEEE Trans. Veh. Technol., vol. 44, no. 2, pp. 318-326, May 1995.

[2] Z. Ying, T. Bolin, V. Plicanic, A. Derneryd, and G. Kristesson, "Diversity antenna terminal evaluation," in Proc. IEEE Int. Symp. on Antennas and Propagation, Jul. 2005, vol. 2A, pp. 375-378.

[3] J. H. Winters, "The diversity gain of transmit diversity in wireless systems with Rayleigh fading," IEEE Trans. Veh. Technol., vol. 47, no. 1, pp. 119-123, Feb. 2001.

[4] M. Karaboikis, C. Soras, G. Tsachtsiris, and V. Makios, "Three branch antenna diversity system on wireless devices using various printed Monopoles," in Proc. IEEE Int. Symp. on Electromagnetic Compatibility, May 2003, vol. 1, pp. 135-138.

[5] M. Kang and M.-S. Alouni, "Capacity of correlated MIMO Rayleigh channels," IEEE Trans. Wireless Commun., vol. 5, no. 1, pp. 143-155, Jan. 2006.

[6] A. Lozano and A. M. Tulino, "Capacity of multiple-Transmit multiple-Receive antenna architectures," IEEE Trans. Inf. Theory, vol. 48, no. 12, pp. 3117-3127, Dec. 2002.

[7] H. Shin and J. H. Lee, "Capacity of multiple-antenna fading channels: Spatial fading correlation, double scattering, and keyhole," IEEE Trans. Inf. Theory, vol. 49, pp. 2636-2647, Oct. 2003.

[8] M. Chiani, M. Z. Win, and A. Zanella, "On the capacity of spatially correlated MIMO Rayleigh-fading channels," IEEE Trans. Inf. Theory, vol. 49, no. 10, pp. 2363-2371, Oct. 2003.

[9] B. Lindmark and M. Nilsson, "On the available diversity gain from different dual-polarized antennas," IEEE J. Sel. Areas Commun., vol. 19, pp. 287-294, Feb. 2001

[10] R. G. Vaughan, "Polarization diversity in mobile communications," IEEE Trans. Veh. Technol., vol. 39, pp. 177-186, Aug. 1990.

[11] R. Vaughan, "Switched parasitic elements for antenna diversity," IEEE Trans. Antennas Propag., vol. 47, no. 2, pp. 399-405, Feb. 1999.

[12] J. F. Valenzuela-Valdés, M. A. García-Fernández, A. M. MartínezGonzález, and D. Sánchez-Hernández, "The role of polarization diversity for MIMO systems under Rayleigh-fading environments," IEEE Antennas Wireless Propag. Lett., vol. 5, pp. 534-536, 2006.

[13] P. Hallbjörner, "The significant of radiation efficiencies when using S-parameters to calculate the received signal correlation from two antennas," IEEE Antennas Wireless Propag. Lett., vol. 4, pp. 97-99, 2005.

[14] K. Ogawa, T. Matsuyoshi, and K. Monma, "An analysis of the performance of a handset diversity antenna influenced by head, hand, and shoulder effects at $900 \mathrm{MHZ}$ : Part I-Effective gain characteristics," IEEE Trans. Veh. Technol., vol. 50, no. 3, pp. 830-844, May 2001.
[15] O. Gasparini, E. de Marinis, and M. Iarossi, "Evaluation of MIMO spatial multiplexing for wireless LAN with channel models from experimental data," presented at the IST Mobile and Wireless Telecommunications Summit, Thessaloniki, Greece, Jun. 17-19, 2002.

[16] S. Loyka and G. Tsoulos, "Estimating MIMO system performance using the correlation matrix approach," IEEE Commun. Lett., vol. 6, no. 1, pp. 19-21, Jan. 2002.

[17] M. Martínez-Vázquez, O. Litschke, M. Geissler, D. Heberling, A. M. Martínez-González, and D. Sánchez-Hernández, "Integrated planar multi-band antennas for personal communications handsets," IEEE Trans. Antennas Propag., vol. 54, no. 2, pp. 384-391, 2006.

[18] K. Meksamoot, M. Krairiksh, and J. Takada, "A polarization diversity PIFA on portable telephone and human body effects on its performance," IEICE Trans. Commun., vol. E84.b, no. 9, pp. 2460-2467, Sep. 2001.

[19] G. F. Pedersen and S. Skjaerris, "Influence on antenna diversity for a handheld phone by the presence of a person," in Proc. 47th IEEE Vehicular Technology Conf., May 1997, vol. 3, pp. 1768-1772.

[20] R. G. Vaughan and J. B. Andersen, "Antenna diversity in mobile communications," IEEE Trans. Veh. Technol., vol. 36, no. 4, pp. 149-172, Nov. 1987.

[21] M. A. Jensen and J. W. Wallace, "A review of antennas and propagation for MIMO wireless communications," IEEE Trans. Antennas Propag., vol. 52, no. 11, pp. 2810-2824, Nov. 2004.

[22] P. S. Kildal, K. Rosengren, J. Byun, and J. Lee, "Definition of effective diversity gain and how to measure it in a reverberation chamber," Microw. Opt. Technol. Lett., vol. 34, no. 1, pp. 56-59, Jul. 2002.

[23] K. Rosengren and P. S. Kildal, "Study of distributions of modes and plane waves in reverberation chamber for characterization of antennas in multipath environment," Microw. Opt. Technol. Lett., vol. 30, pp. 386-391, Sep. 2001.

[24] K. Rosengren and P. S. Kildal, "Radiation efficiency, correlation, diversity gain and capacity of a six-monopole antenna array for a MIMO system: Theory, simulation and measurement in reverberation chamber," Proc. Inst. Elect. Eng. on Microwaves, Antennas and Propagation, vol. 152, no. 1, pp. 7-16, Feb. 2005.

[25] G. J. Foschini and M. J. Gans, "On limits of wireless communications in a fading environment when using multiple antennas," Wireless Personal Commun., vol. 6, no. 3, pp. 311-335, Mar. 1998.

[26] I. E. Telatar, "Capacity of multi-antenna Gaussian channels," Eur Trans. Telecommun., vol. 10, no. 6, pp. 585-596, Nov./Dec. 1999.

[27] J. Yang, J. Carlsson, P. S. Kildal, and C. Carlsson, "Calculation of self-impedance and radiation efficiency of a dipole near a lossy cylinder with arbitrary cross section by using the moment method and a spectrum of two dimensional solutions," Microw. Opt. Technol. Lett., vol 32, no. 2, pp. 108-112, Jan. 2002.

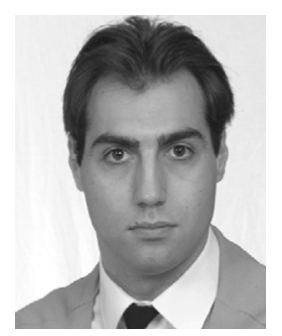

Juan F. Valenzuela-Valdés was born in Marbella, Spain. He received the Degree in telecommunications engineering from the Universidad de Malaga, Spain, in 2003. He is currently working toward the Ph.D. degree at the Universidad Politécnica de Cartagena, Cartagena, Spain

In 2004, he worked at CETECOM, Malaga, in the same year he joined the Department of Information Technologies and Communications, Universidad Politécnica de Cartagena, Spain. In 2007, he joined EMITE Ingeniería SLNE, Espinardo, Spain, as Head of Research. His current research areas cover MIMO communications, reverberation chambers and SAR measurements.

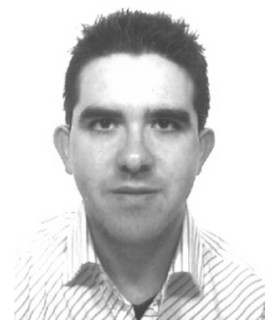

Miguel Á. García-Fernández was born in Cartagena, Spain. He received the Degree in telecommunications engineering from the Universidad Politécnica de Cartagena, in 2005, where he is currently working toward the Ph.D. degree.

In 2005, he joined the Department of Information Technologies and Communications, Universidad Politécnica de Cartagena. His current research areas cover SAR measurements and thermoregulatory processes due to electromagnetic field exposure. 


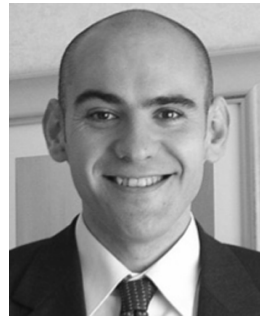

Antonio M. Martínez-González received the Dipl.-Ing. degree in telecommunications engineering from the Universidad Politécnica de Valencia, Spain, in 1998 and the Ph.D. degree from the Universidad Politécnica de Cartagena, Cartagena, Spain, in 2004

From 1998 to September 1999, he was employed as Technical Engineer at the Electromagnetic Compatibility Laboratory, Universidad Politécnica de Valencia, where he developed assessment activities and compliance certifications with European directives related with immunity and emissions to electromagnetic radiation from diverse electrical, electronic and telecommunication equipment. Since September 1999, he has been an Assistant Lecturer at the Universidad Politécnica de Cartagena. His research interest is focused on electromagnetic dosimetry and radioelectric emissions.

Dr. Martínez-González's research work was awarded the Spanish National Prize in 1999 from Foundation Airtel and the Colegio Oficial de Ingenieros de Telecomunicación de España for the Best Final Project on Mobile Communications.

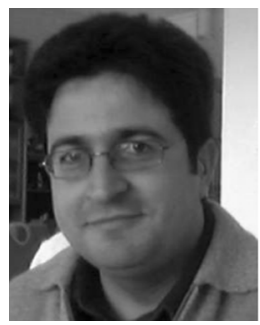

David A. Sánchez-Hernández (M'00-SM’06) received the Dipl.-Ing. degree in telecommunications engineering from the Universidad Politécnica de Valencia, Valencia, Spain, in 1992 and the Ph.D. degree from King's College, University of London, London, U.K., in 1996.

From 1992 to 1994 , he was employed as a Research Associate for The British Council-CAM at King's College London. where he worked on active and dual-band microstrip patch antennas. In 1994, he was appointed EU Research Fellow at
King's College London, working on several joint projects at 18, 38, and 60 $\mathrm{GHz}$ related to printed and integrated antennas on GaAs, microstrip antenna arrays, sectorization and diversity. In 1997, he returned to the Universidad Politécnica de Valencia, where was co-leader of the Antennas, Microwaves and Radar Research Group and the Microwave Heating Group. In early 1999, he received the Readership from the Universidad Politécnica de Cartagena, and was appointed Vice Dean of the School for Telecommunications Engineering and Leader of the Microwave, Radiocommunications and Electromagnetism Engineering Research Group. In late 1999, he was appointed Vice Chancellor for Innovation and Technology Transfer at the Universidad Politécnica de Cartagena, Cartagena, and a member of several foundations and societies for promotion of R\&D in the Autonomous Region of Murcia, Spain. In May 2001, he was appointed Official Advisor in Technology Transfer and member of The Industrial Advisory Council of the Autonomous Government of the Region of Murcia, in Spain. In May 2003, he was appointed Head of the Communications and Signal Theory Department. He has published over 35 scientific papers and over 70 conference contributions, and is a reviewer of several international journals. His current research interests encompass all aspects of the design and application of printed multiband antennas for mobile communications, electromagnetic dosimetry issues and MIMO techniques for wireless communications.

Dr. Sánchez-Hernández is a Chartered Engineer (C.Eng.). He is a Fellow of the Institution of Engineering and Technology (IET) Fellow, London, U.K. He is an Ampere Board member, CENELEC TC106X member, and is the recipient of the R\&D J. Langham Thompson Premium, awarded by the Institution of Electrical Engineers (currently the Institution of Engineering and Technology), as well as other national and international awards. 